\title{
Biomechanical assessment and clinical analysis of different intramedullary nailing systems for oblique fractures
}

\author{
J. A. Alierta ${ }^{a}$, M. A. Pérez ${ }^{b}$, B. Seral-García ${ }^{c}$, J. M. García-Aznar
}

${ }^{a}$ Ministry of Defense, Spain

${ }^{\mathrm{b}}$ Aragón Institute of Engineering Research (I3A), Department of Mechanical Engineering Universidad de Zaragoza, Zaragoza, Spain

${ }^{c}$ Orthopaedic Surgery.

Lozano Blesa University Hospital. Zaragoza. Spain

Submitted: 2015

Corresponding author:

Dr. José Manuel García-Aznar

Mechanical Engineering, Universidad de Zaragoza

Ed. Betancourt-Campus Río Ebro, C/María de Luna 50018 Zaragoza, Spain

Tel: 0034976762796

Fax: 0034976762670

e-mail: jmgaraz@unizar.es 


\section{Abstract}

The aim of this study is to evaluate the fracture union or non-union for a specific patient that presented oblique fractures in tibia and fibula, using a mechanistic-based bone healing model. Normally, this kind of fractures can be treated through an intermedullary nail using two possible configurations that depends on the mechanical stabilization: static and dynamic. Both cases are simulated under different fracture geometries in order to understand the effect of the mechanical stabilisation on the fracture healing outcome. The results of both simulations are in good agreement with previous clinical experience. From the results, it is demonstrated that the dynamization of the fracture improves healing in comparison with a static or rigid fixation of the fracture. This work shows the versatility and potential of a mechanistic-based bone healing model to predict the final outcome (union, non-union, delayed union) of realistic $3 \mathrm{D}$ fractures where even more than one bone is involved.

\section{Keywords:}

Bone healing, finite element predictions, design of fixators, oblique fractures, multiple bone fragments. 


\section{Introduction}

Bone fractures are one of the most common orthopaedic problems, where bone loses the continuity and the typical transfer of loads is disrupted between the bone fragments. Although many different factors regulate how these bone fragments heal (such as genetic, cellular and biochemical factors, blood supply, neural and hormonal regulation, age) (Goodship et al.,1993; Hadjiargyrou et al.,1998; Einhorn,2005; Jagodzinski and Krettek,2007; Marsell and Einhorn,2011), it is the local mechanical and geometrical environment which mainly direct the regeneration also regulating other biological phenomena (Gómez-Benito et al.,2005; Checa and Prendergast,2009; Wehner et al.,2010).

In clinical orthopaedics, different kinds of fractures can occur, existing different systematic classifications (Neer,2002; Garnavos et al.,2012; Brorson,2013). One of the most typical bone fractures in long bones are oblique fractures in the diaphysis. In these cases, the fracture line runs obliquely to the shaft of the bone. Its treatment is identical to the used in other transverse fractures; however there is not a profound knowledge of the influence of the geometry of the oblique fracture (angle and location) on the healing process. In this sense, computational models could be an interesting alternative to understand its regeneration. Nevertheless, most of Finite Element (FE)-based computational models have only focused on the simulation of transverse fractures (Lacroix and Prendergast,2002a; Gómez-Benito et al.,2005; Shefelbine et al.,2005; García-Aznar et al.,2007; Checa and Prendergast,2009; Byrne et al.,2011; Simon et al.,2011). Only the works of Loboa et al. (2001) and Comiskey et al. (2013) have analysed the healing phenomenon in oblique fractures, but not analysing the simultaneous healing of more than a fracture in different bones, being this event something that happens frequently in clinical cases. Probably, this kind of fractures has not been studied by means of Finite Element Analysis (FEA) due to the complexity of using current bone healing algorithms in real 3D geometries. However, recently, Alierta et al.(2014) have presented a novel mechanistic phenomenological approach for modelling bone fracture healing specially 
adequated for the simulation of realistic 3D geometries. Actually, this model allows the simulation of bone fracture healing for realistic bone fracture geometries (oblique, comminuted, spiral and compound). The model also enables to study and analyse the improvement of bone healing depending on the use of different fixation systems, such as, locking plates, nails, external fixators and intramedullary screws. In particular, in this work we are going to use this mechanistic phenomenological bone healing model (Alierta et al.,2014) to simulate one clinical case where the fibula and tibia of one patient were obliquely fractured and where they were treated by means of an intramedullary nail system distinguishing between an static and dynamical configurations.

\section{Material and methods}

The bone fracture gap is modelled through the incorporation of interface elements that connect the two fracture ends simulating the discontinuity in the displacement field between bone fragments. These interface elements were originally presented by Alierta et al. (2014) to simulate bone discontinuities that occur in bone fractures. In addition, this model is able to consider bone healing, recovering bone continuity in the bone tissue. In the present work, we apply this mechanistic interface model to study a clinical case, where fractures occur in the diaphysis of tibia and fibula. Next the main features necessary to understand and follow the implications of the model are presented.

\subsection{Interface finite element model used to predict healing outcome of bone fractures}

To model the fracture gap, cohesive elements (Fig. 1a) are used to connect the fracture ends (García-Aznar et al.,2009). The thickness of these elements corresponds to the dimension of the gap fracture. 
Accordingly, the constitutive law of these interface elements is directly established in terms of traction versus separation law ${ }^{1}$, and depends on:

(1) A set of three model parameters in each direction i ( $i=n$, normal; $s$ and $t$, tangential): $K_{0 i}$ (linear stiffness in a completely healed fracture gap along the direction i), $\varepsilon_{0 i}$ (maximum strain in the corresponding direction at the linear region), $\varepsilon_{c i}$ (maximum strain allowed in the corresponding direction). These parameters characterize the mechanical behavior of the healing interface (See Fig. 1b-c).

(2) A state variable $\alpha$, designated as union degree, that quantifies the degree of healing or union. In particular, $\alpha$ is defined as the ratio between the current value of the stiffness $K_{i}$ in any direction $\mathrm{i}$ and the one corresponding to the fully bonded interface in that direction $K_{0 i}$ (Fig. $1 \mathrm{~b}-\mathrm{c}$ ). With this definition, $\alpha$ is automatically normalized between 0 and 1 , corresponding $\alpha=0$ to a completely non-union or malunion and $\alpha=1$ to a totally successful bone healing .

For the compression traction $\left(\varepsilon_{n}<0\right)$, we assume a linear behaviour with respect to the associated strain, until $\varepsilon_{n}$ reaches the value -1 , which means that the fracture ends are in contact. If lower values of $\varepsilon_{n}$ are reached the simulation will be aborted because no penetration is allowed.

The state variable $\alpha$ can decrease or grow. The union degree, $\alpha$ decreases $(\dot{\alpha}<0)$ because the limit strain $\left(\varepsilon_{\alpha}\right)$ is reached (for example, due to a very high load). If this fact occurs during the healing process, the healing will be impaired or at least delayed. In this situation, the final value selected for the union degree is the minimum value associated to each direction $\left(\alpha=\min \left\{\alpha_{s}, \alpha_{t}, \alpha_{n}\right\}\right)$.

\footnotetext{
${ }^{1}$ As a first approximation in this work, we have assumed a linear relationship between traction and separation in the fractured gap, although a non-linear law could be easily implemented. 
The union degree, $\alpha$, can increase due to bone healing, therefore it is calculated as the addition of two contributions: $\alpha=\alpha_{c}+\alpha_{b}$, where $\alpha_{c}$ reflects the recovery of the mechanical properties of the fracture gap mainly due to the formation of cartilage, and $\alpha_{b}$ reflects the recovery of the mechanical properties of the fracture gap due to bone formation.

In order to estimate the temporal evolution of $\alpha\left(\dot{\alpha}=\dot{\alpha}_{c}+\dot{\alpha}_{b}\right)$, we propose a regulatory law based on three different healing zones (adapted from Claes et al. (1999)) which are established as a function of the compression, $\varepsilon_{n}$, and the shear strains $\left(\varepsilon_{\text {shear }}=\sqrt{\varepsilon_{s}^{2}+\varepsilon_{t}^{2}}\right)$ of each interface element (Fig. 1d). A maturation time for cartilage $\left(M_{c}\right)$ is defined and once it is reached, the value of $\alpha_{c}$ will grow exponentially (Fig. 1e) until it reaches the maximum value $\left(\alpha_{c \max }\right)$. In a similar way, once the maturation time for bone $\left(M_{b}\right)$ is reached, the value of $\alpha_{b}$ will grow linearly (Fig. 1f) until it reaches the maximum value $\left(\alpha_{\mathrm{bmax}}\right)$ at the total healing time $\left(t_{h}\right)$.

The values of the model parameters, according to Alierta et al. (2014), are shown in Table 1.

The healing algorithm was numerically implemented in an Abaqus user subroutine (UMAT) and all the finite element analyses (FEA) were carried out in Abaqus v.6.11 (Dassault Systèmes Simulia Corp.) by means of the interface elements available in Abaqus $v 6.11$ and known as COH3D6. In any case, the reader is referred to the work of Alierta et al. (2014) for further details.

\subsection{Clinical case: Oblique fractures in the fibula and the tibia}

A patient (male, $1.70 \mathrm{~m}$ and $76 \mathrm{~kg}$ ) presented a fracture in the right fibula and tibia (Fig. 2), which was treated with an intramedullary nail $\left(8 \mathrm{~mm}\right.$ diameter cannulated EXPERT ${ }^{\mathrm{TM}}$ nail of $330 \mathrm{~mm}$ length).

The 3D FE model of the fractured tibia and fibula is constructed (Fig. 3a), with the help of the software Mimics (Materialise), from a set of CT scans (distance between slices of $3 \mathrm{~mm}$ ). With 
regard to the bone, a distinction between cortical and trabecular parts has been made. The geometry obtained is meshed in 3-matic (Materialise). The element size used (approximately 1 $\mathrm{mm}$ ) is inside the asymptotic region of convergence and represents a good trade off between numerical accuracy and computational cost.

The fracture gaps of the fibula and the tibia are modelled using the software Abaqus (Dassault Systèmes Simulia Corp.) by 439 interface elements (linear wedges) and the rest of the model consists of 347289 linear tetrahedral linear elements. The mechanical properties of the bone and gap tissue are assumed to be linear elastic, isotropic and homogeneous. Titanium alloy (Ti$6 \mathrm{Al}-7 \mathrm{Nb})$ is the material used for nails and bolts (EXPERT $T^{T M}$ ). In Table 2, we show the exact material properties that have been used for the simulations, except for the mechanical properties of the gap tissue, which are regulated by the interface finite element model presented in the section 2.1. and Table 1.

Two different proximal locking configurations of the Expert ${ }^{\mathrm{TM}}$ nail are simulated: 1) the proximal locking screws are located in the circular hole (Fig. 3b) to achieve, according to the nail's manufacturer specifications, direct healing, in which the bone heals without apparent callus and skipping the intermediate steps of tissue differentiation and resorption (Perren,2002), and 2) the proximal locking screw is located in the upper zone of the slotted hole (Fig. 3b) to achieve, according to the results of the clinical treatment, indirect healing that consists of the sequential steps of tissue differentiation, resorption of the surfaces of the fracture and joining the fracture fragments by the callus (Perren,2002). In the first configuration (static configuration), the fixation is more rigid because the proximal screw moves jointly with the nail, however, in the second configuration (dynamical configuration), the proximal screw moves freely in the slotted hole doing the fixation less rigid. 
The geometrical properties of the nail are taken from mechanical drawing data, obtained from a direct measure of a nail, using a three-dimensional coordinate measuring machine (DEA Gamma 0102). The union between the nail and the bone was modelled as a surface-surface contact with a friction coefficient of 0.3 (Rancourt et al.,1990). The $4 \mathrm{~mm}$ diameter locking bolts ( 2 distal and 1 proximal) are modelled by 26 linear beam elements, which nodes are joined to the nail's and bone's nodes placed next to them, using MPC (Multi-point constraint).

A vertical load of $760 \mathrm{~N}$ (corresponding to a single leg stance of a patient of approximately 76 $\mathrm{kg}($ Wehner et al.,2010)) is applied at the proximal condyles in the four cases. The knee contact force is not uniformly shared between the compartments of the tibial plateau: $60 \%$ of the load is borne by the medial condyle and $40 \%$ by the lateral condyle (Duda et al.,2001; Perez et al.,2010) (Fig. 3c). The nodes of the distal part of the tibia are constrained so that, the rigidbody motions are avoided (Fig. 3d).

\section{Results}

The temporal evolution of the union degree, $\alpha$, is evaluated for both the static and the dynamic configurations (Fig. 4). Only one curve is represented because the same evolution of the union degree is predicted for the fracture gap of the tibia and fibula. The union degree pattern is represented in Fig. 5 for both configurations and for the tibia and fibula. Almost the same variation of the union degree is observed. The value of this variable at the end of the consolidation time, $t_{h}=60$ days (Klein et al.,2004), is completely in concordance with the expected results by the clinicians: $\alpha=\alpha_{b \max }=0.7$ for direct ossification (static configuration) and $\alpha=\alpha_{\max }=1$ for indirect ossification (dynamic configuration) (Fig. 5). The temporal evolution of the interfragmentary movements (IFM) in the compressive (Fig. 6) and shear (Fig. 7) directions for both configurations are shown in both fracture sites. In both configurations, the IFM is 
slightly lower in the fibula than in the tibia. As it can be observed, the recovery of the mechanical properties of the fracture gap, i.e. the reduction of the interfragmentary movements, is more evident for the dynamic configuration (Fig. 8).

The temporal evolution of the maximum Von Mises stress in the intramedullary nail for both configurations is shown in Fig. 9. Although the initial values were very similar, the stress was reduced in a more noticeable way in the dynamic configuration. In both cases, this stress was initially concentrated in the fracture zone and in the distal locking bolts, as can be observed in Fig. 10. Nevertheless, in the final stage of healing ( $t_{\text {healing }}=60$ days) the stress distribution differs from the static to the dynamic configuration; in the static configuration the stress concentration is located, as initially, in the fracture zone and in the distal locking bolts, whereas in the dynamic configuration the stress concentration is located only in the fracture zone (Fig. 10).

\section{Discussion}

In the present study we apply a mechanistic phenomenological bone healing model (Alierta et al.,2014) to a clinical case where fractures of the fibula and tibia simultaneously heal. The main advantage of the Finite Element-based approach is that it allows simulating in 3D the healing of two or more fractures sites or different bone fragments at the same time. This approach is very advantageous to simulate complex bone fractures and to understand the impact that different orthopaedic treatments can have on the healing phenomenon and in the wholeorgan.

In particular, in this work we investigate the role of two different intramedullary designs (static and dynamic) for the treatment of oblique fractures in tibia and fibula corresponding to a clinical case. As far as authors know only two previous FE-based computational works, Loboa et al. (2001) and Comiskey et al. (2013), have studied stresses and strains patterns around the 
site of an oblique fracture but using a mechanobiological 2D approach where specific tissue differentiation is considered. However, they did not analyse the effect of the fracture geometry and location on the pattern healing in realistic 3D geometries. Nevertheless, several mechanobiological approaches (Loboa et al.,2001; Lacroix and Prendergast,2002a; Shefelbine et al.,2005; Andreykiv et al.,2008; Isaksson et al.,2008; Checa and Prendergast,2009; Wehner et al.,2010; Reina-Romo et al.,2011; Simon et al.,2011) have been previously used to study in 2D and 3D the influence of local mechanical conditions on biological events that regulate the temporal and spatial evolution of the different ossification mechanisms that occur during healing on the fracture gap in transversal fractures.

But these mechanolobiological theories have not been used for modelling complex fracture geometries, due to the difficulty of working with these geometries. Modelling bone fractures by means of interface elements is an adequate alternative that is a helpful tool to model complicate bone fractures.

In this work, we use this novel tool to study the effect of different concepts of intramedullary nail systems (static and dynamic) on the treatment of a double oblique fracture that occurs in the tibia and the fibula corresponding to a clinical case.

This clinical case analysis allows demonstrating that dynamization of the fracture could improve healing in comparison to rigid fixation of the fracture. A more rigid fixation promotes direct ossification (Fig. 4), therefore, the quality of regenerated bone in direct healing cannot be always guaranteed and therefore a new fracture may occur. But, a more flexible fixation allows a higher, but controlled, instability of the fracture gap region. This promotes indirect healing which is the most common in clinical treatments, since the high quality of the regenerated tissue (Marsell and Einhorn,2011). Although this effect was also observed by previous studies of the literature (Claes et al.,1995; Bishop et al.,2006) in sheep experiments, 
this modelling work allows to understand the dynamization effect in a patient with specific oblique fractures.

Furthermore, the numerical results here presented are qualitatively similar to those available in clinical experience: successful indirect healing with the dynamic configuration according to the clinical outcome, and direct healing with the static configuration according to the nail's manufacturer specification.

Nevertheless, we have to keep in mind that we have assumed some simplifications in our model that need further analysis. Firstly, we have considered a constant load during all the healing time as other previous works (Lacroix and Prendergast,2002b). In particular, due to the lack of data of the load borne by the patient during the healing process a single leg stance load was considered (Wehner et al.,2010) without including muscle forces. However, there are other works (Duda et al.,2001; Byrne et al.,2011) that indicate that the inclusion of other muscles cause a considerable unloading of the fracture gap. Secondly, the vast majority of bone fracture repair occurs by secondary fracture healing, where a multistage process of tissue regeneration stabilises the bone with an external callus and repairs the fracture via endochondral ossification. A limitation of our phenomenological model is that we do not exactly model the callus growth around the fracture site, although we take into account the effect of the callus geometry on the fracture site stiffness. An additional limitation is that while our phenomenological approach simulates the healing of oblique fractures, we have not been able to illustrate the exact tissue healing differentiation patterns. Mechanobiological models present many limitations for modelling complex fracture geometries; however they are more powerful in representing the tissue healing differentiation patterns. Finally, another limitation is related with the fracture gap size. The interface elements used for the model implementation can only be used with fractures where the dimension of the gap is thin enough to consider it negligible with respect to the overall dimensions of the bone fracture. 
Therefore, this approach is not valid for large defects where other kind of models should be considered.

Despite these simplifications, the phenomenological approach here proposed presents practical implications that allow to create FE-based model specific for one patient taking into account the exact geometries of the bone fractures. The combination of this methodology with others based for example on artificial neuronal networks (Garijo et al.,2014) to estimate patient-specific loads will allow in a future to help the surgeons to apply patient-specific treatments. Actually, given the bone geometry corresponding to a patient and its fracture characteristics as the input data, we will be able to estimate the stiffness and the optimal position of the fixator for this specific patient.

Therefore, in order to conclude, this work presents a practical application of the previously developed phenomenological model (Alierta et al.,2014), showing its versatility to simulate realistic complicated 3D bone fractures in which even more than one bone are involved. The satisfactory use of the previously cited model to choose the most suitable configuration to stabilize one specific patient fracture has also been demonstrated throughout this work.

\section{Conflict of interest statement}

The authors have no conflict of interest.

\section{Acknowledgements}

The research leading to these results has received funding from the European Union's Seventh Framework Programme (FP7/2007-2013) under Grant agreement no. 286179 (CAD-BONE) and the Spanish Ministry of Economy and Competitiveness through research project DPI 201453401-C2-1-R. 


\section{References}

Alierta, J. A., M. A. Perez,J. M. Garcia-Aznar, 2014. An interface finite element model can be used to predict healing outcome of bone fractures. J. Mech. Behav. Biomed. Mater. 29: 328-338.

Andreykiv, A., F. v. Keulen, P. J. Prendergast, 2008. Simulation of fracture healing incorporating mechanoregulation of tissue differentiation and dispersal/proliferation of cells. Biomech. Model. Mechanobiol. 7: 443-461.

Bishop, N. E., M. v. Rhinjn, I. Tami, R. Corveleijn, E. Schneider,K. Ito, 2006. Shear does not necessarily inhibit bone healing. Clin. Orthop. Relat. Res. 443: 307-314.

Brorson, S., 2013. Fractures of the proximal humerus. Acta Orthop. Suppl. 84(351): 1-32.

Byrne, D. P., D. Lacroix,P. J. Prendergast, 2011. Simulation of Fracture Healing in the Tibia: Mechanoregulation of Cell Activity Using a Lattice Modeling Approach. J. Orthop. Res. October: 1496-1503.

Claes, L. E.,C. A. Heigele, 1999. Magnitudes of local stress and strain along bony surfaces predict the course and type of fracture healing. J. Biomech. 32: 255-266.

Claes, L. E., H.-J. Wilke, P. Augat, S. Rübenacker,K. J. Margevicius, 1995. Effect of dynamization on gap healing of diaphyseal fractures under external fixation. Clin. Biomech. 10(5): 227-234.

Comiskey, D., B. J. MacDonald, W. T. McCartney, K. Synnott,J. O'Byrne, 2013. Predicting the external formation of callus tissues in oblique bone fractures: idealised and clinical case studies. Biomech. Model. Mechanobiol. 12(6): 1277-1282.

Checa, S.,P. Prendergast, 2009. A mechanobiological model for tissue differentiation that includes angiogenesis: a lattice-based modeling approach. Ann. Biomed. Eng. 37(1): 129-145.

Duda, G. N., F. Mandruzzato, M. Heller, J. Goldhahn, R. Moser, M. Hehli, L. Claes, N. P. Haas, 2001. Mechanical boundary conditions of fracture healing: borderline indications in the treatment of unreamed tibial nailing. J. Biomech. 34(5): 639-650.

Einhorn, T. A., 2005. The science of fracture healing. J. Orthop. Trauma 19(10): S4-S6.

García-Aznar, J. M., J. H. Kuiper, M. J. Gómez-Benito, M. Doblaré,J. B. Richardson, 2007. Computational simulation of fracture healing: Influence of interfragmentary movement on the callus growth. J. Biomech. 40: 1467-1476.

García-Aznar, J. M., M. A. Pérez,P. Moreo, 2009. Modelling of Interfaces in Biomechanics and Mechanobiology. Computer modeling in engineering \& sciences : CMES 48(3): 271-301.

Garijo, N., J. Martínez, J. M. García-Aznar,M. A. Pérez, 2014. Computational evaluation of different numerical tools for the prediction of proximal femur loads from bone morphology. Comput. Methods. Appl. Mech. Engrg. 268: 437-450.

Garnavos, C., N. K. Kanakaris, N. G. Lasanianos, P. Tzortzi, R. M. West, 2012. New classification system for long-bone fractures supplementing the AO/OTA classification. Orthopedics 35(5): e709-719.

Gómez-Benito, M. J., J. M. García-Aznar, J. H. Kuiper,M. Doblaré, 2005. Influence of fracture gap size on the pattern of long bone healing: a computational study. J. Theor. Biol. 235: 105-119.

Goodship, A. E., P. E. Watkins, H. S. Rigby,J. Kenwright, 1993. The role of fixator frame stiffness in the control of fracture healing. An experimental study. J. Biomech. 26(9): 1027-1035.

Hadjiargyrou, M., K. McLeod, J. Ryaby, C. Rubin, 1998. Enhancement of fracture healing by low intensity ultrasound. Clin. Orthop. Relat. Res. 355(S): 216-229.

Isaksson, H., C. C. v. Donkelaar, R. Huiskes, K. Ito, 2008. A mechano-regulatory bone-healing model incorporating cell-phenotype specific activity. J. Theor. Biol. 252: 230-246.

Jagodzinski, M., C. Krettek, 2007. Effect of mechanical stability on fracture healing - an update. Injury 38(S1): S3-10. 
Klein, P., M. Opitz, H. Schell, W. R. Taylor, M. O. Heller, J.-P. Kassi, F. Kandziora,G. N. Duda, 2004. Comparison of unreamed nailing and external fixation of tibial diastasesmechanical conditions during healing and biological outcome. J. Orthop. Res. 22: 10721078.

Lacroix, D.,P. J. Prendergast, 2002a. A mechano-regulation model for tissue differentiation during fracture healing: analysis of gap size and loading. J. Biomech. 35: 1163-1171.

Lacroix, D.,P. J. Prendergast, 2002b. Three-dimensional Simulation of Fracture Repair in the Human Tibia. Comput. Methods Biomech. Biomed. Engin. 5(5): 369-376.

Loboa, E. G., G. S. Beaupré,D. R. Carter, 2001. Mechanology of initial pseudarthrosis formation with oblique fractures. J. Orthop. Res. 19: 1067-1072.

Marsell, R.,T. Einhorn, 2011. The biology of fracture healing. Injury 42(6): 551-555.

Neer, C. S., 2nd, 2002. Four-segment classification of proximal humeral fractures: purpose and reliable use. J. Shoulder Elbow Surg. 11(4): 389-400.

Niinomi, N., 1998. Mechanical properties of biomedical titanium allows. Materials Science and Engineering A243: 231-236.

Perez, M. A., P. Fornells, M. Doblare,J. M. Garcia-Aznar, 2010. Comparative analysis of bone remodelling models with respect to computerised tomography-based finite element models of bone. Comput. Methods Biomech. Biomed. Engin. 13(1): 71-80.

Perren, S. M., 2002. Evolution of the internal fixation of long bone fractures. J. Bone Joint Surg. Br. 84-B(8): 1093-1110.

Rancourt, D., A. Shirazi-Adl, G. Drouin,G. Paiement, 1990. Friction properties of the interface between porous-surfaced metals and tibial cancellous bone. J. Biomed. Mater. Res. 24(11): 1503-1519.

Reina-Romo, E., M. J. Gómez-Benito, J. Domínguez, F. Niemeyer, T. Wehner, U. Simon,L. E. Claes., 2011. Effect of the fixator stiffness on the young regenerate bone after bone transport: Computational approach. J. Biomech. 44: 917-923.

Shefelbine, S. J., P. Augat, L. Claes,U. Simon, 2005. Trabecular bone fracture healing simulation with finite element analysis and fuzzy logic. J. Biomech. 38: 2440-2450.

Simon, U., P. Augat, L. Claes, M. Utz, 2011. A numerical model of the fracture healing process that describes tissue development and revascularisation. Comput. Methods Biomech. Biomed. Engin. 14(1): 79-93.

Wehner, T., L. Claes, F. Niemeyer, D. Nolte,U. Simon, 2010. Influence of the fixation stability on the healing time- $A$ numerical study of a patient-specific fracture healing process. Clin. Biomech. 25: 606-612. 


\section{List of figures}

Fig. 1 Constitutive model: (a) cohesive elements, (b) shear traction, (c) normal traction, (d) healing zones, (e) temporal evolution of $\alpha_{c}$ and (f) temporal evolution of $\alpha_{b}$. (with permission from Alierta et al. (2014))

Fig. 2 (a) Radiograph of the fibula and tibia fracture with the fixator and (b) dimensions of the fractures $(\mathrm{mm})$

Fig. 3 (a) FE model, (b) possible locations of the proximal locking bolt, (c) distribution of the applied load and (d) displacement boundary conditions.

Fig. 4 Temporal evolution of the maximum union degree, $\alpha$, for both configurations.

Fig. 5 Temporal evolution of the union degree, $\alpha$, for both configurations in the fracture sites.

Fig. 6 Evolution of the interfragmentary movement in the compressive direction (IFMc) in the fracture sites throughout the healing process for both configurations.

Fig. 7 Evolution of the interfragmentary movement in the shear direction $\left(\mathrm{IFM}_{\mathrm{sh}}\right)$ in the fracture sites throughout the healing process for both configurations.

Fig. 8 Maximum interfragmentary movement $(a)$ in the compressive direction (IFM $M_{c}$ ) and (b) in the shear direction (IFM $\mathrm{Fh}_{\mathrm{sh}}$ ) for the static and dynamic configurations.

Fig. 9 Time evolution of the Von Mises Stress in the intramedullary nail for the static and the dynamic configurations.

Fig. 10 Distribution of the Von Mises Stress (MPa) in the intramedullary nail for the static and the dynamic configurations throughout the healing process. 


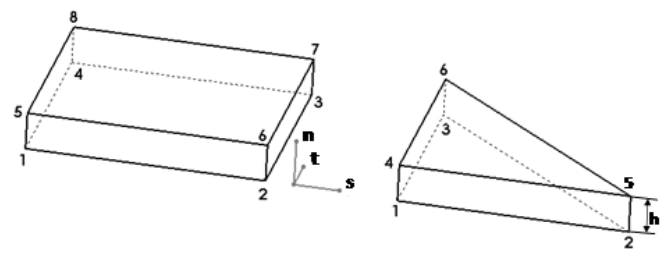

b

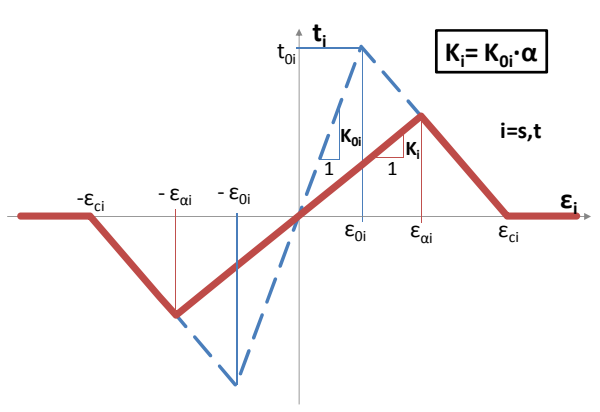

C

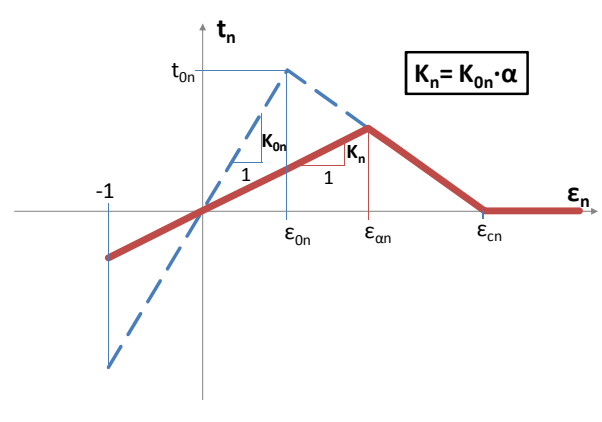

d

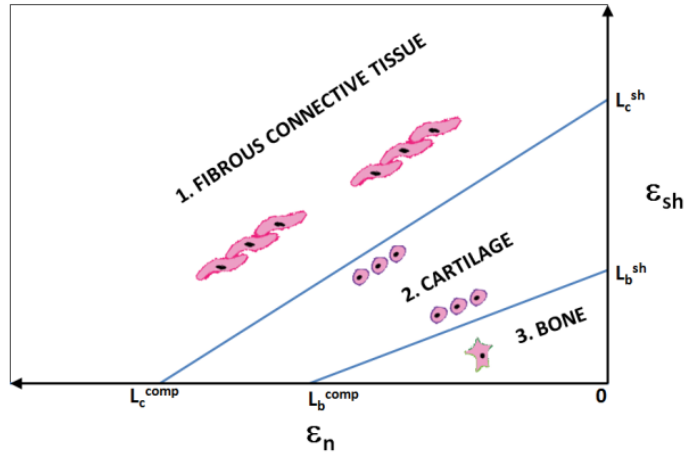

e

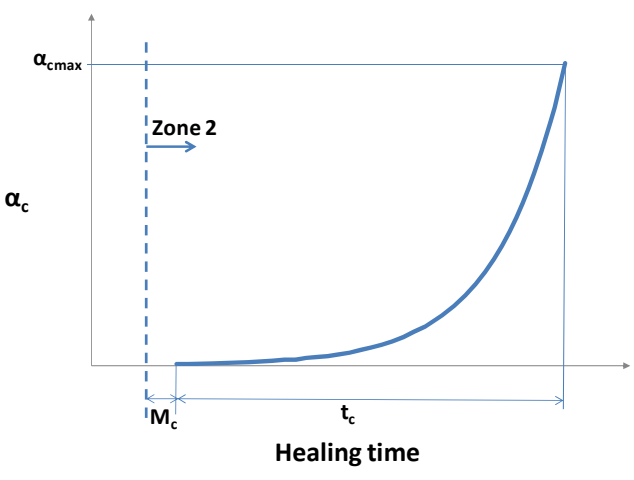

$f$

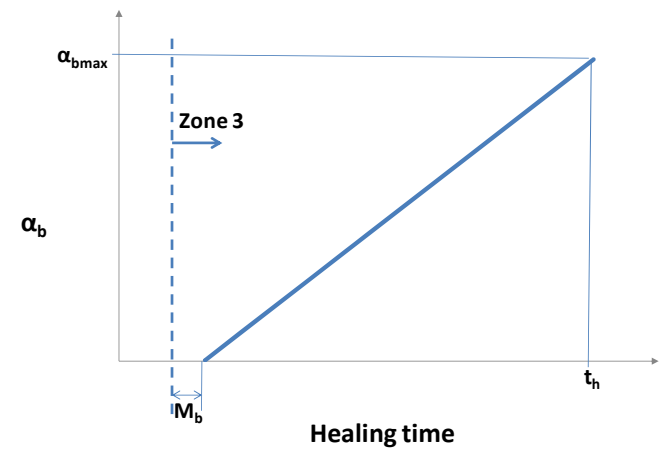

Fig. 1 Constitutive model: (a) cohesive elements, (b) shear traction, (c) normal traction, (d) healing zones, (e) temporal evolution of $\alpha_{c}$ and (f) temporal evolution of $\alpha_{b}$. (with permission from Alierta et al. (2014)). 
a

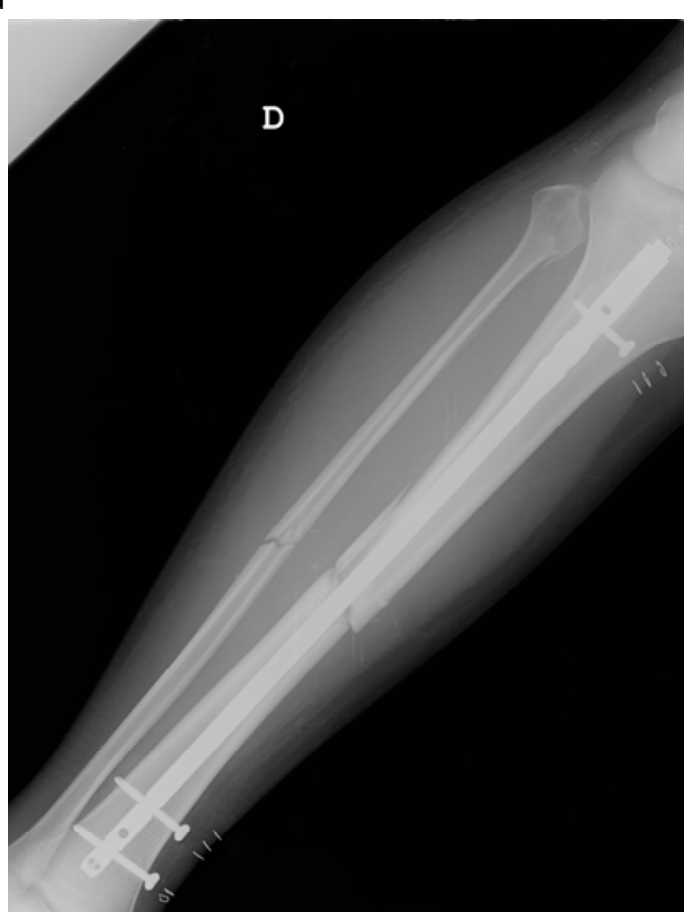

b

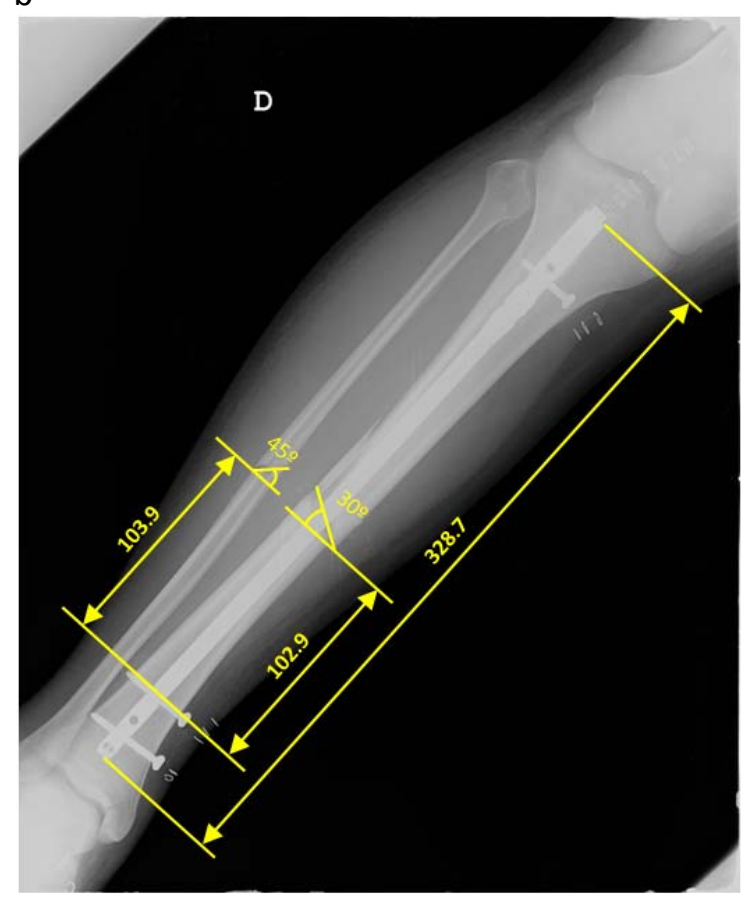

Fig. 2 (a) Radiograph of the fibula and tibia fracture with the fixator and (b) dimensions of the fractures $(\mathrm{mm})$. 
a

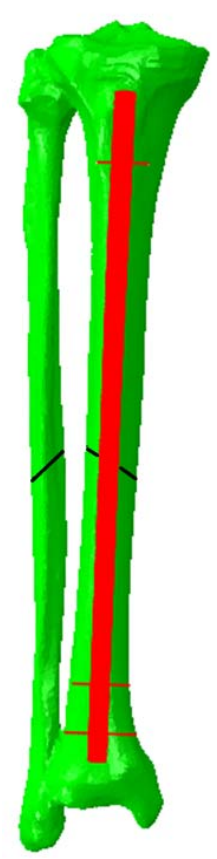

C

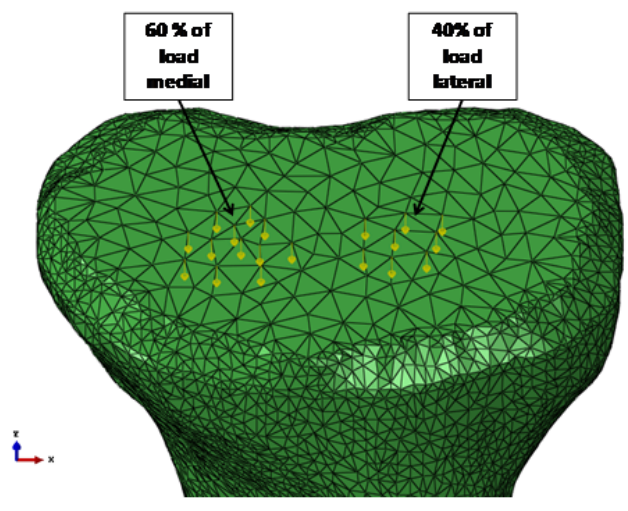

b

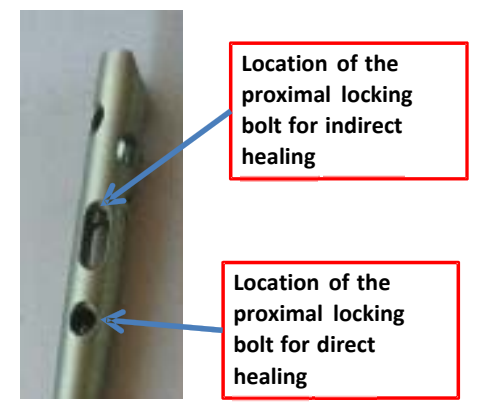

d

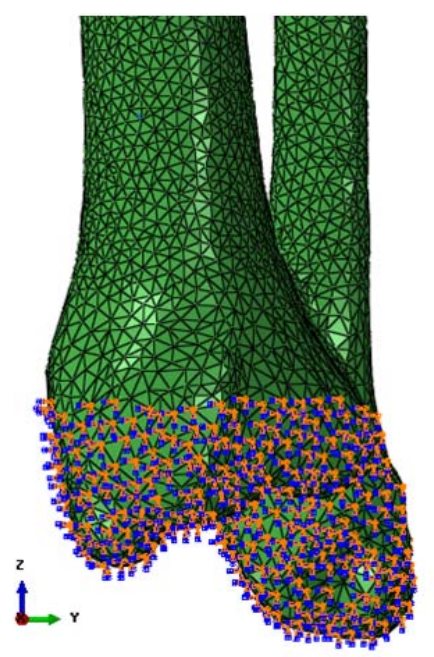

Fig. 3 (a) FE model, (b) possible locations of the proximal locking bolt, (c) distribution of the applied load and (d) displacement boundary conditions. 
Union degree, $\alpha$

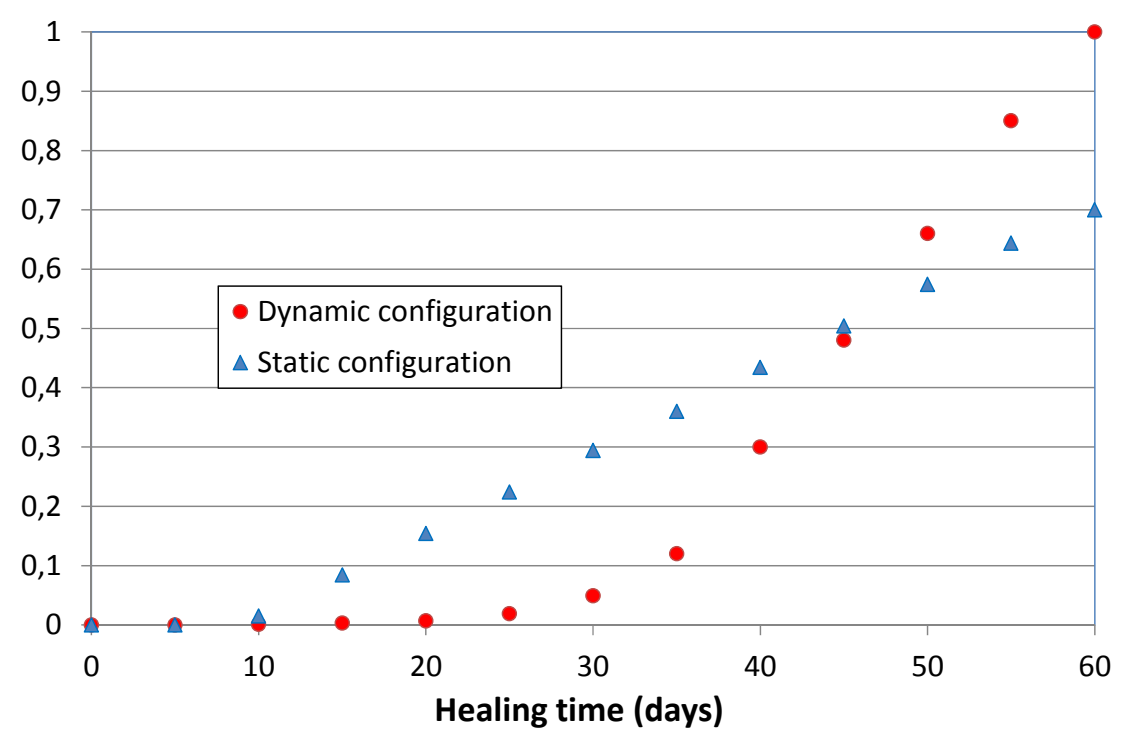

Fig. 4 Temporal evolution of the maximum union degree, $\alpha$, for both configurations. 


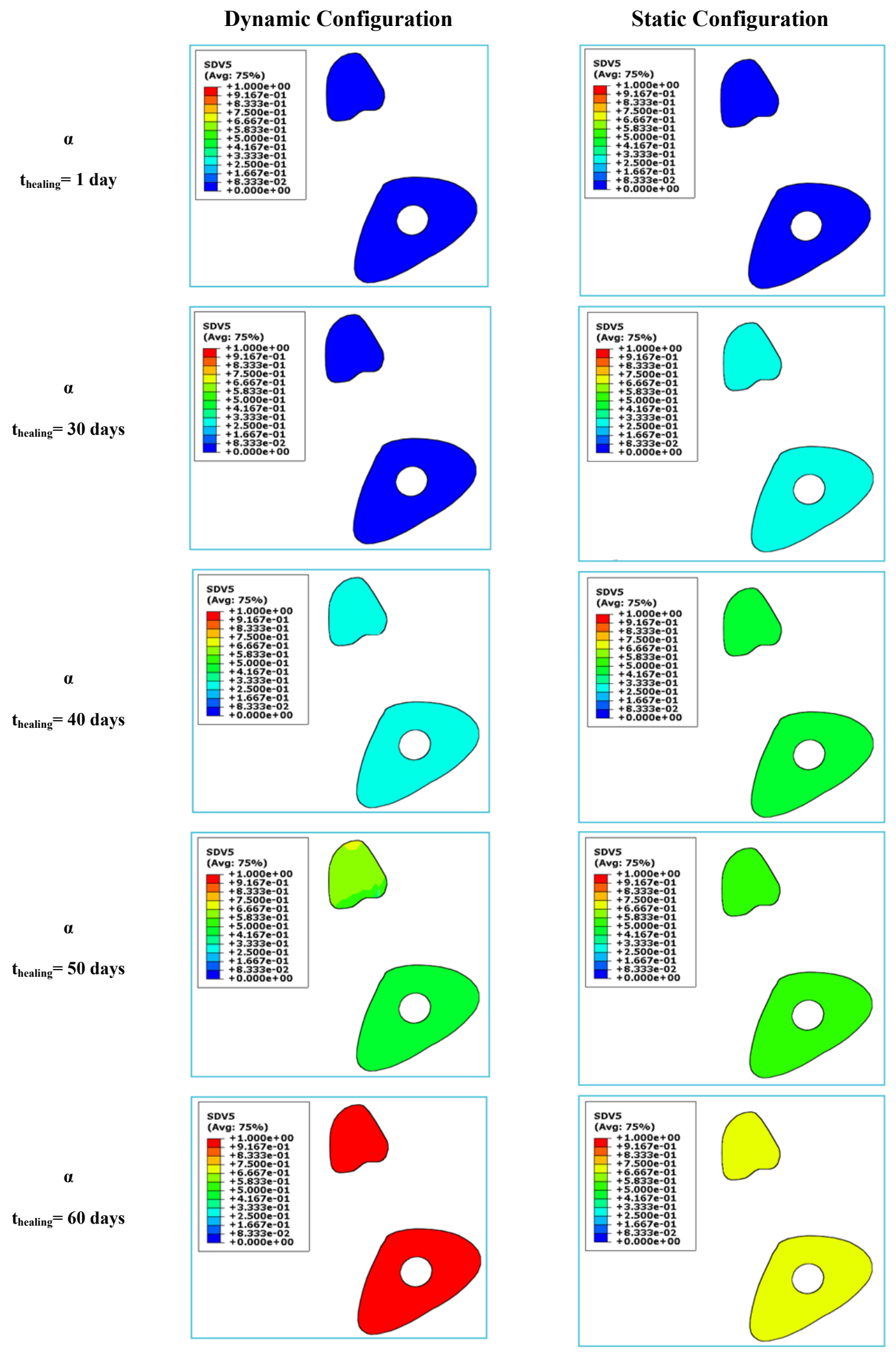

Fig. 5 Temporal evolution of the union degree, $\alpha$, for both configurations in the fracture sites. 


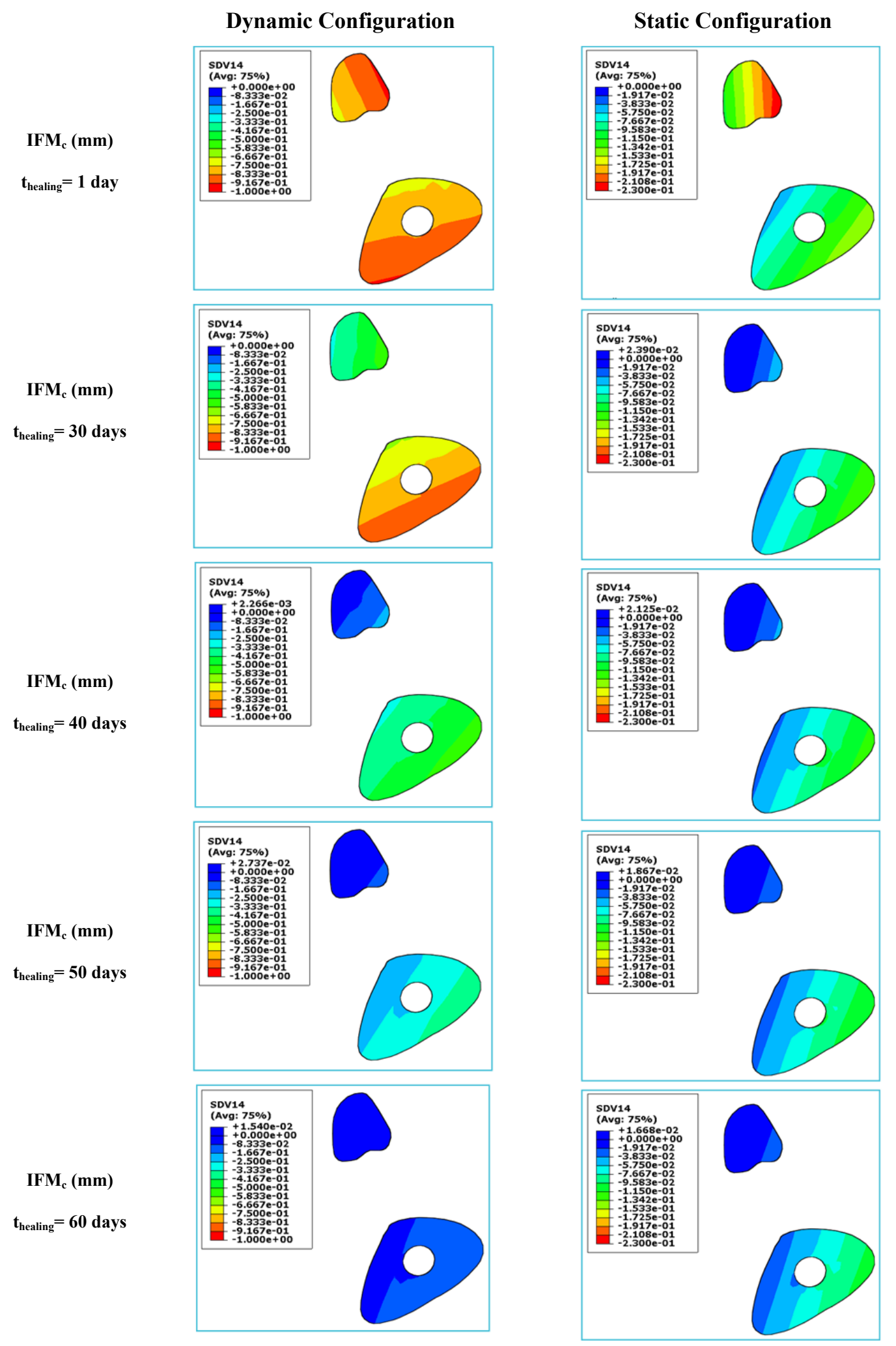

Fig. 6 Evolution of the interfragmentary movement in the compressive direction ( $\left(\mathrm{FM}_{\mathrm{c}}\right.$ ) in the fracture sites throughout the healing process for both configurations. 


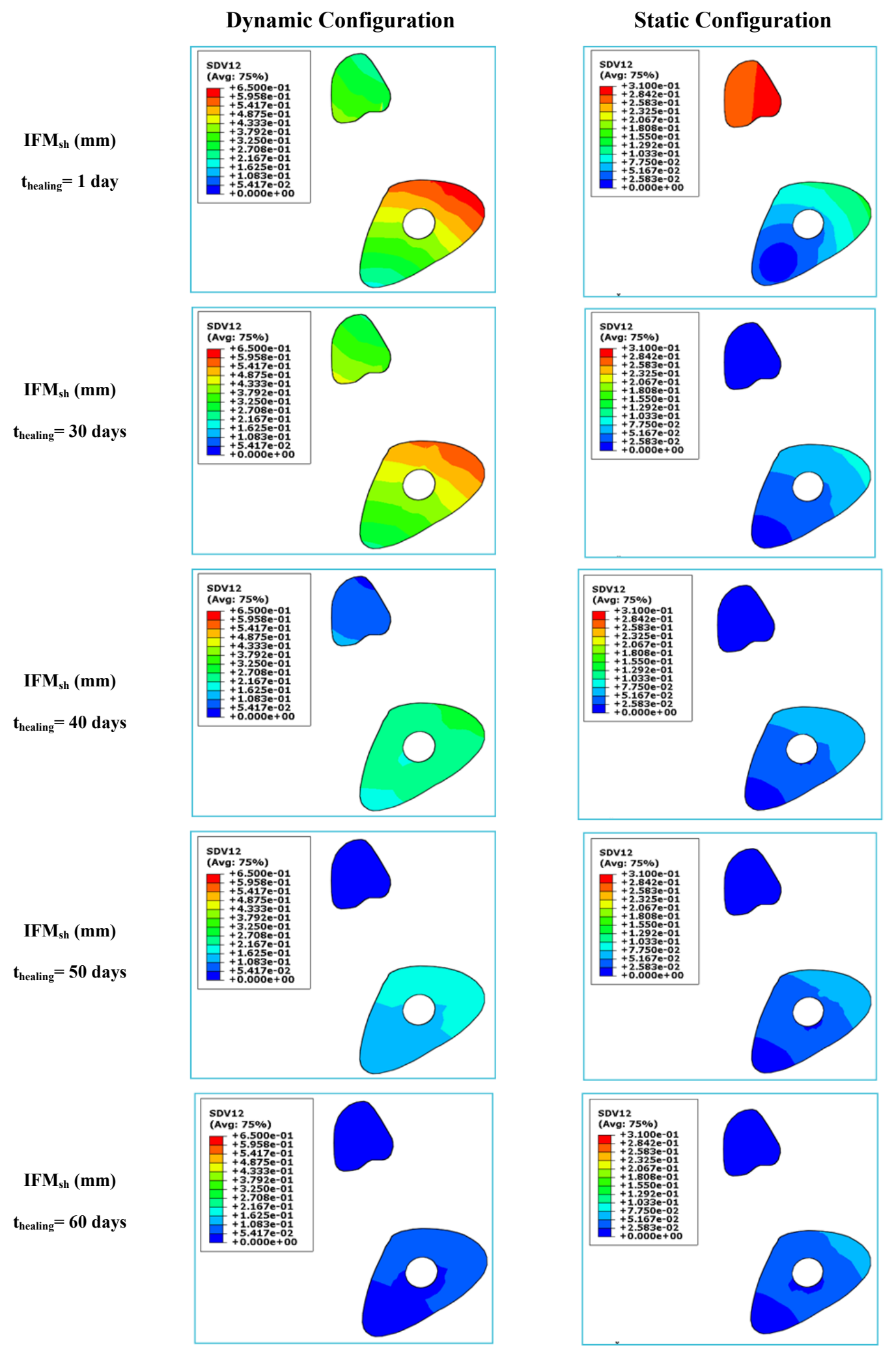

Fig. 7 Evolution of the interfragmentary movement in the shear direction (IFM $M_{s h}$ ) in the fracture sites throughout the healing process for both configurations. 
$\operatorname{IFM}_{\mathrm{c}}(\mathrm{mm})$

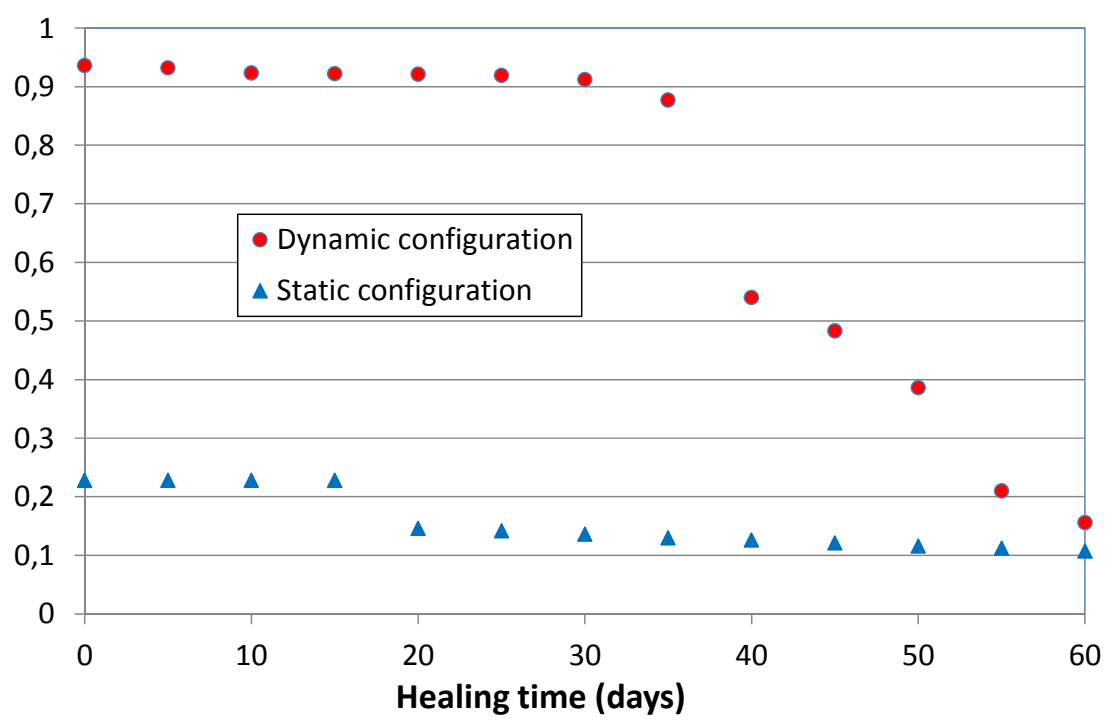

b

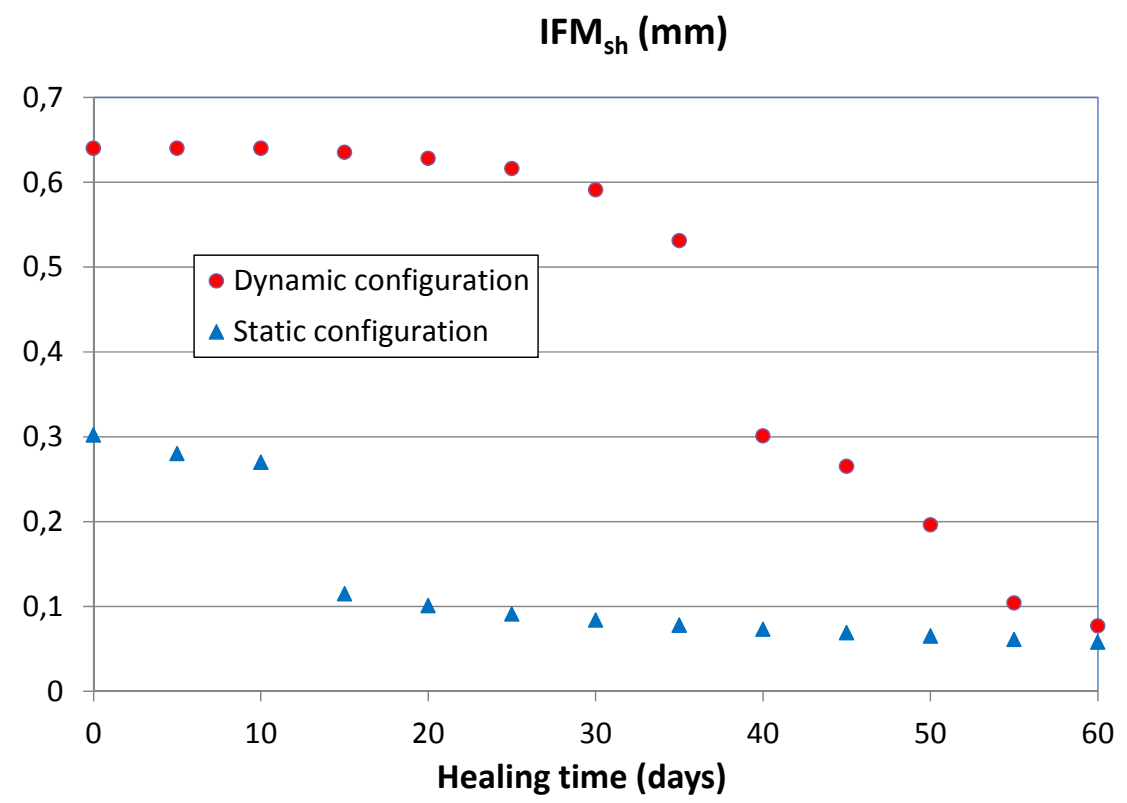

Fig. 8 Maximum interfragmentary movement (a) in the compressive direction (IFMc) and (b) in the shear direction $\left(\mathrm{IFM}_{\mathrm{sh}}\right)$ for the static and dynamic configurations. 


\section{Von Mises Stress (MPa)}

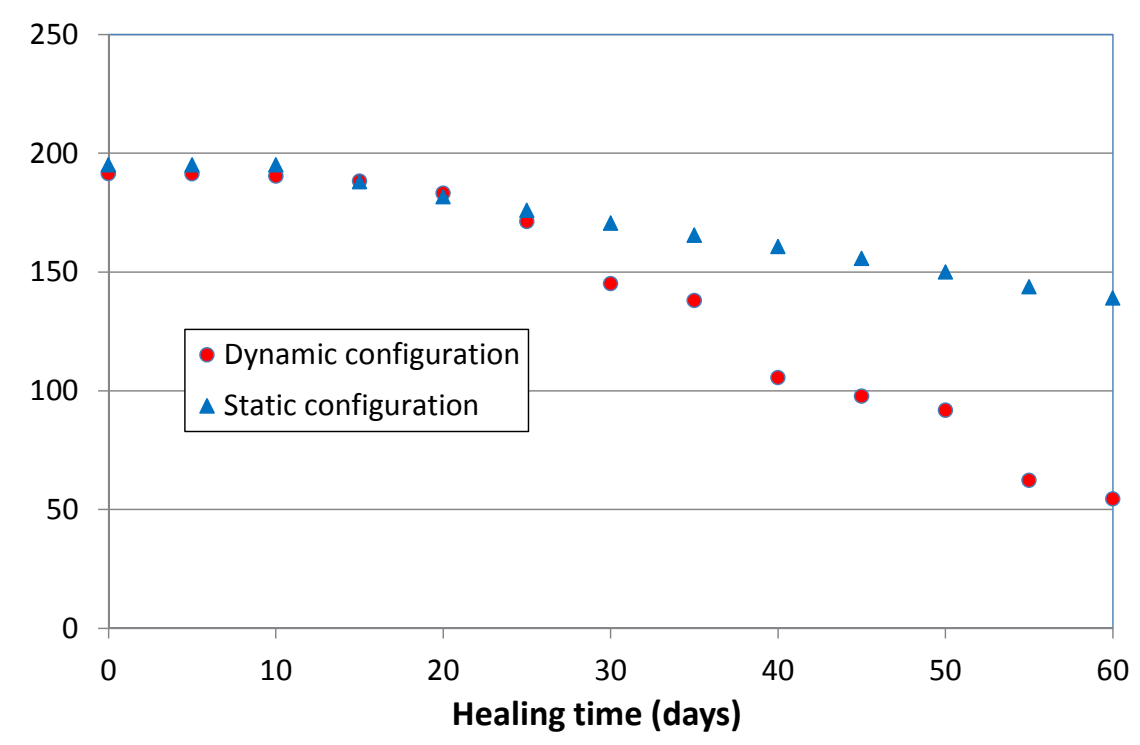

Fig. 9 Time evolution of the Von Mises Stress in the intramedullary nail for the static and the dynamic configurations. 


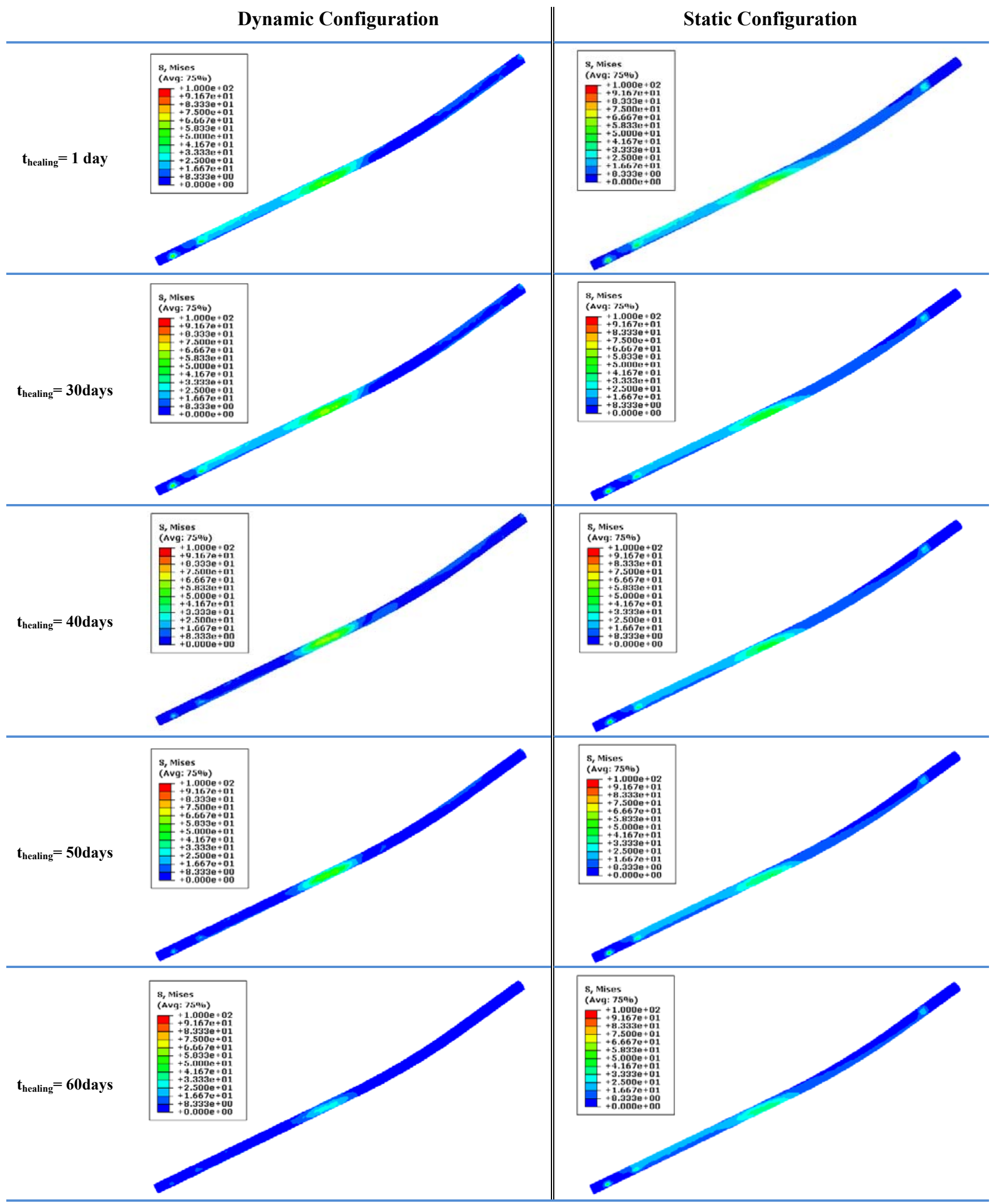

Fig. 10 Distribution of the Von Mises Stress (MPa) in the intramedullary nail for the static and the dynamic configurations throughout the healing process. . 


\section{List of tables}

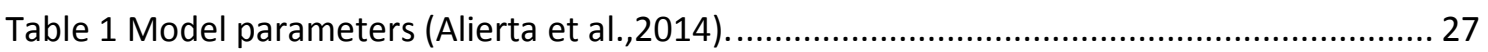

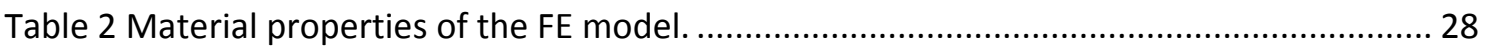


Table 1 Model parameters (Alierta et al.,2014).

\begin{tabular}{lll}
\hline Parameter & & Value \\
\hline$M_{c}$ & Maturation time for cartilage (days) (see Fig. 1e) & 4 \\
$M_{b}$ & Maturation time for bone (days) (see Fig. 1f) & 10 \\
$\alpha_{c m a x}$ & Maximum value of $\alpha_{c}$ (see Fig. 1e) & 0.3 \\
$\alpha_{b m a x}$ & Maximum value of $\alpha_{\mathrm{b}}$ (see Fig. 1f) & 0.7 \\
$t_{c}$ & Cartilage formation time (days) (see Fig. 1e) & 36 \\
$t_{h}$ & Total healing time (days) (see Fig. 1f) & 60 \\
$L_{c}^{c o m p}$ & Compression strain limit for cartilage formation (see Fig. 1d) & 0.6 \\
$L_{b}^{c o m p}$ & Compression strain limit for bone formation (see Fig. 1d) & 0.25 \\
$L_{c}^{s h}$ & Shear strain limit for cartilage formation (see Fig. 1d) & 0.25 \\
$L_{b}^{s h}$ & Shear strain limit for bone formation (see Fig. 1d) & 0.15 \\
$K_{o i}(i=n, s, t)$ & Initial linear stiffness (MPa) & 50 \\
$\varepsilon_{0 i}(i=n, s, t)$ & Maximum strain at the linear region (see Fig. 1b-c) & 0.3 \\
$\varepsilon_{c i}(i=n, s, t)$ & Maximum allowed strain (see Fig. 1 b-c) & 1 \\
\hline & & \\
\hline
\end{tabular}


Table 2 Material properties of the FE model.

\begin{tabular}{lll}
\hline Material & Young's modulus (MPa) & Poisson's coefficient \\
\hline Cortical bone $^{\mathrm{a}}$ & 17000 & 0.3 \\
Trabecular bone $^{\mathrm{a}}$ & 100 & 0.2 \\
Steel & 210000 & 0.3 \\
Ti-6Al-7 $\mathrm{Nb}^{\mathrm{b}}$ & 114000 & 0.3 \\
\hline
\end{tabular}

${ }^{a}$ Duda et al. (2001)

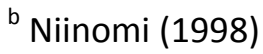

